\title{
Análise da fórmula SRK/T no cálculo de lente intra-ocular em cães portadores de catarata
}

\author{
[Analysis of the SRK/T formula for calculation of intra-ocular lens in dogs carrying cataract] \\ T.P. Peixoto, J.J.T. Ranzani, C.V.S. Brandão, A.C.L. Rodrigues \\ Faculdade de Medicina Veterinária e Zootecnia - UNESP \\ Caixa Postal 560 \\ 18618-000 - Botucatu, SP
}

\begin{abstract}
RESUMO
Foram utilizados 20 cães de raças e idades variadas, machos e fêmeas, portadores de catarata e não diabéticos, os quais foram submetidos ao exame oftálmico. Posteriormente, realizaram-se mensurações oculares empregando-se um ecobiômetro ultra-sônico (ultra-sonografia modo-A) para o cálculo do poder dióptrico da lente intra-ocular por meio da fórmula SRK/T. O comprimento axial médio foi de $19,94 \pm 1,12 \mathrm{~mm}$. Todos os animais foram submetidos à facoemulsificação extracapsular. A lente calculada foi implantada no transoperatório da cirurgia de catarata, obtendo-se média de $37,33 \pm 3,05 \mathrm{D}$. A avaliação pós-cirúrgica do erro refracional aos 60 dias de pós-operatório, pela retinoscopia, com a utilização da

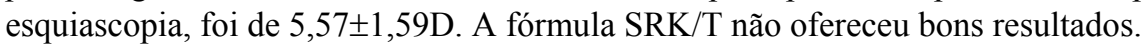

Palavras-chave: cão, emétrope, catarata, fórmula SRK/T, erro refracional

\begin{abstract}
Twenty males and females non-diabetic dogs of different breeds and ages underwent ophthalmic examination because they presented catarats. Ocular measurements were performed by echobiometry $(A-$ scan ultrasound) for intraocular lens power calculation using the SRK/T formula. The obtained mean axial length was $19.94 \pm 1.12 \mathrm{~mm}$. All animals were submitted to extracapsular phacoemulsification; the mean intraocular lens power implanted was 37.33 \pm 3.05 . At 60 days postoperative, the refractional error assessed via retinoscopy was $5.57 \pm 1.59 \mathrm{D}$. The SRK/T formula did not offer good results.
\end{abstract}

Keywords: dog, emetropic, cataract, SRK/T formula, refractional error

\section{INTRODUÇÃO}

A catarata, como termo médico, compreende um grupo de desordens oculares manifestadas por opacidade das fibras do cristalino ou de sua cápsula. Possui tamanhos, formas, causas e graus de progressão variados (Narfström e Bjerkås, 2001; Slatter, 2001). Uma variedade de agentes terapêuticos tem sido estudada, tanto em homens como em cães, relativamente às ações de prevenção, retardo ou reversão sem melhora efetiva (Davidson e Nelms, 1999; Narfström e Bjerkås, 2001). O tratamento para a catarata consiste na sua remoção cirúrgica (Fischer, 1989). Atualmente, a técnica de escolha para remoção da catarata é a facoemulsificação (Bistner, 1992; Glover e Constantinescu, 1997; Rodrigues, 2004).

A disponibilidade, desde 1984, do uso de lentes intra-oculares (LIO) projetadas para uso em cães, tem permitido reabilitação óptica do olho de cães após a cirurgia para remoção da catarata (Davidson et al., 1993). Outro método é por meio do uso de LIO humanas em piggyback, ou polipseudoafacia (Hull et al., 1999; Eleftheriadis, 2001).

O cálculo do poder refrativo da LIO, determinado antes do procedimento cirúrgico, é

Recebido em 5 de novembro de 2007

Aceito em 21 de outubro de 2008

E-mail: quim@fmvz.unesp.br 
essencial e de extrema importância para atingir um estado emétrope (Erkin et al., 1999), apesar de Ranzani et al., 2008 terem constatado em cães normais, uma tendência à hipermetropia, ao efetuarem retinoscopia com luz em faixa. No passado, as LIO eram calculadas utilizando-se fórmulas analíticas baseadas na óptica de Gaussian. Basicamente, este cálculo depende da mensuração, automática ou manual, do poder refrativo corneal (raio de curvatura da córnea) e do comprimento axial do olho (distância entre a córnea e a retina) (Gaiddon et al., 1991; Erkin et al., 1999; Murphy et al., 2002; Preussner et al., 2002).

As fórmulas de primeira geração (Colenbrander, Fyodorov, Hoffer, van der Heijde, Thijssen e Binkhorst), além do comprimento e da ceratometria, requeriam um valor constante da profundidade de câmara anterior (PCA), para posicionar a LIO. Baseavam-se na fórmula de "Gernet", com um sistema de duas lentes; a córnea e a lente pseudofácica (LIO) focando imagens na retina. As de segunda geração foram propostas no século passado, quando a constante de PCA foi substituída por uma que variava baseada no comprimento axial (Hoffer, 1993; Lagenbucher e Seitz, 2004).

Fórmulas de terceira geração variam a sua PCA em função do comprimento axial e da curvatura corneal do paciente. A PCA da fórmula Holladay é a soma da altura e espessura corneal $(0,56 \mathrm{~mm})$, com a distância do plano da íris até o plano central da LIO, o que é denominado de fator do cirurgião (FC) (Hoffer, 1993; Jaffe et al., 1997). Retzlaff et al. (1990) desenvolveram uma nova fórmula de terceira geração, a SRK/T. Essa fórmula é semelhante à Holladay, mas, ao invés do FC, apresenta uma constante-A (constante calculada empiricamente para cada tipo de LIO) para produzir um valor a ser adicionado à altura corneal da fórmula de Fyodorov e resultar em uma PCA; ela também usa um diferente índice refrativo corneal (Hoffer, 1993). Estas fórmulas mais complexas (Holladay, Holladay 2, SRK/T e HofferQ), que combinam cálculos analíticos e ajustes empíricos para os diferentes parâmetros, também requerem um fator para a espessura da retina (Preussner et al., 2002).

A ultra-sonografia de modo-A é uma apresentação acústica unidimensional utilizando ondas de som não focalizadas para produzir uma série linear de picos ecóicos, que são representados como espinhos verticais da linha basal. O tempo entre os picos ecóicos pode ser convertido em distância, baseando-se na velocidade do som no meio onde os ecos são recebidos (Davidson e Nelms, 1999).

A ultra-sonografia de modo-A, para fins biométricos, é baseada em um transdutor piezoelétrico oscilatório, que gera disparos curtos e intermitentes de ondas sonoras numa única direção, através dos tecidos. Essas ondas de alta freqüência são refletidas, parcialmente, pelas interfaces dos tecidos, apresentando diferentes densidades e níveis de independência acústica, que resultam em ecos pulsáteis recebidos pelo transdutor original. Os ecos são filtrados, amplificados e gravados pelo aparelho. Os tempos de intervalos dos interpulsos podem ser medidos por fotografias ou métodos eletrônicos (Schiffer et al., 1982; Davidson e Nelms, 1999).

Com o presente estudo, objetivou-se analisar a fórmula SRK/T para o cálculo do poder da LIO e verificar sua indicação para o olho de cães.

\section{MATERIAL E MÉTODO}

Foram utilizados 20 cães de raças e idades variadas, machos e fêmeas, portadores de catarata e não diabéticos. Esses animais foram distribuídos em dois grupos: G1 (grupo de 10 animais submetidos à facoemulsificação extracapsular com implantação de lentes intraoculares em piggyback) e G2 (grupo de 10 animais submetidos à facoemulsificação sem a implantação de lente intra-ocular).

Os animais foram submetidos ao exame oftálmico de teste da lágrima de Schirmer, tonometria de aplanação, oftalmoscopia direta e prova da fluoresceína. Aqueles que apresentaram alterações oculares ou sistêmicas que inviabilizavam a realização da cirurgia foram descartados.

Foram feitas as avaliações ultra-sonográficas em três momentos (M1, M2, M3) após a tranqüilização química e instilação de colírio anestésico $^{1}$. O exame consistiu de seis mensurações consecutivas das seguintes

${ }^{1}$ Anestalcon, Alcon Laboratórios do Brasil Ltda. - São Paulo, Brasil. 
estruturas oculares: comprimento axial do bulbo ocular e distância entre a córnea e a cápsula anterior do cristalino.

As mensurações citadas acima foram obtidas automaticamente pela ultra-sonografia de modoA, empregando-se um ecobiômetro ultra-sônico ${ }^{2}$ e técnica de imersão em metilcelulose $2 \%{ }^{3}$ descrita por Giers e Epple (1990). A sonda de $10 \mathrm{mhz}$ foi alinhada com eixo óptico do olho, e o aparelho calibrado para as velocidades de 1,526 $\mathrm{m} / \mathrm{s}$ para o humor aquoso e vítreo e $1,710 \mathrm{~m} / \mathrm{s}$ para o cristalino. Apenas em casos excepcionais, as mensurações foram feitas manualmente devido a erros na leitura no modo automático. Esse processo também impossibilitou a aferição da profundidade de câmara anterior após o procedimento cirúrgico.

A mensuração da curvatura corneal (ceratometria) foi processada com a utilização de um autoceratômetro ${ }^{4}$, efetuando-se cinco aferições consecutivas no olho operado e no contralateral, em M1 (antes do procedimento cirúrgico), M2 (30 dias pós-cirurgia) e M3 (60 dias pós-cirurgia).

O cálculo do poder dióptrico da LIO foi feito pelo próprio aparelho, por meio da fórmula SRK/T, utilizando-se a média das aferições do comprimento axial e da ceratometria. A constante da lente intra-ocular (constante-A) de 118,0 , utilizada na fórmula, foi fornecida pelo fabricante. $\mathrm{O}$ poder dióptrico obtido foi dividido entre duas lentes, seguindo o cálculo a seguir: primeira LIO (localizada mais anteriormente) igual a $60 \%$ do poder total da LIO; e segunda LIO (localizada mais posteriormente) igual a $40 \%$ do poder total da $\mathrm{LIO}$, mais $3 \mathrm{D}$.

A extração de catarata, após a incisão em córnea clara, procedeu-se com o aparelho de facoemulsificação ${ }^{5}$, seguida da implantação de duas lentes intra-oculares de silicone dobrável ${ }^{6}$, em piggyback, com utilização de injetor ${ }^{7}$. Foi realizado o fechamento da incisão de $3,2 \mathrm{~mm}$ com

\footnotetext{
${ }^{2}$ Echoscan Model US-800, Nidek Co. - Japão.

${ }^{3}$ Metilcelulose AV, Ophthalmos - SãoPaulo, Brasil.

${ }^{4}$ KM-500. Nidek Co. - Japão.

${ }^{5}$ AMO DIPLOMAX ${ }^{\mathrm{TM}}$, AMO Advanced Medical Optics São Paulo, Brasil.

${ }^{6}$ AMO PhacoFlex II, SI 40 NB, AMO Advanced Medical Optics - São Paulo, Brasil.

${ }^{7}$ The Unfolder Silver Series, Allergan - Irvine, EUA.
}

dois a três pontos simples e separados, utilizando-se fio mononáilon $10-0^{8}$, os quais foram sepultados.

A retinoscopia, com utilização do retinoscópio halógeno de faixa ${ }^{9}$ das réguas de esquiascopia $^{10}$, que permitem a avaliação do erro refracional, foi executada nos animais operados aos 30 (M2) e 60 (M3) dias de pós-operatório.

Para a realização da retinoscopia, precedida da dilatação pupilar com colírio de cloridrato de tropicamida $1 \%{ }^{11}$, o manguito do retinoscópio foi fixado em sua posição mais baixa (efeito de espelho plano) e posicionado a, aproximadamente, $67 \mathrm{~cm}$ do paciente. Quando a faixa e o reflexo se moveram na mesma direção e sem nenhuma lente no aparato refratário, a refração correspondeu a uma das anomalias de refração: hipermetropia, emetropia ou miopia menor que 1,5D; se o reflexo se moveu em direção oposta, o erro da miopia foi considerado maior que $1,5 \mathrm{D}$.

Como as variáveis apresentaram distribuição normal e homogeneidade de variâncias, foi utilizada a análise de perfil nos casos em que houve comparação de grupos e momentos, e de grupos e olhos, e o teste t para a comparação de grupos. O nível de significância considerado foi de $5 \%$.

\section{RESULTADOS}

Os valores pré-cirúrgicos da profundidade da câmara anterior ( $\mathrm{mm}$ ) dos animais do G1 foram semelhantes estatisticamente aos do G2 (Tab. 1; $\mathrm{P}>0,05)$. O comprimento axial do olho dos animais do G1 com catarata foi semelhante ao do G2 (Tab. 2; P>0,05); portanto, cães de olho pequeno. O maior comprimento axial no G1 foi de $21,01 \mathrm{~mm}$ e o menor $17,42 \mathrm{~mm}$. O G2 apresentou um animal com um olho de $22,76 \mathrm{~mm}$.

\footnotetext{
${ }^{8}$ Ethicon, Johnson \& Johnson Ltda. - São José dos Campos, Brasil.

${ }^{9}$ Retinoscópio halógeno de faixa $\mathrm{n}^{\circ} 18200$ de $3,5 \mathrm{v}$, Welch Allyn - Ontário, Canadá

${ }^{10}$ Par de Réguas de Esquiascopia L2 Convexa e Côncava, Luneau Ophtalmologie - Charters, França.

${ }^{11}$ Tropinom, Genom - Embu-Guaçu, Brasil.
} 
Tabela 1. Médias e desvio-padrão pré-cirúrgicos da profundidade da câmara anterior ( $\mathrm{mm}$ ) em cães, segundo o grupo

\begin{tabular}{ccccc} 
Grupo & Olho esquerdo & Olho direito & Média & $\begin{array}{c}\text { Análise de perfil } \\
\text { P-valor }\end{array}$ \\
\hline G1 & $3,74 \pm 0,98$ & $3,68 \pm 0,6$ & $3,71 \pm 0,79$ & 0,073 \\
G2 & $4,37 \pm 0,86$ & $4,19 \pm 0,73$ & $4,28 \pm 0,78$ & 0,565 \\
\hline Média & $4,06 \pm 0,95$ & $3,94 \pm 0,7$ & $3,99 \pm 0,82$ & 0,775 \\
\hline
\end{tabular}

G1: cães submetidos à facoemulsificação com implante de lentes intra-oculares em piggyback, G2: cães submetidos à facoemulsificação.

Não houve diferença entre os olhos $(\mathrm{P}>0,05)$ direito e esquerdo.

Quanto à ceratometria (Tab. 3), não houve diferença entre grupos dentro de momentos e entre momentos dentro de grupo. Após a aplicação dos dados na fórmula SRK/T, obtiveram-se os valores de poder da LIO. A média no grupo a receber o implante foi de $37,32 \pm 3,0 \mathrm{D}$ (olhos operados) e no G2, uma LIO mais fraca, $34,64 \pm 2,88 \mathrm{D}$. Observou-se que, quanto maiores os comprimentos axiais e a profundidade das câmaras anteriores, menor será a dioptria da lente. A maior lente calculada do G1 foi de 40,5D, e a menor de 33D.

Tabela 2. Média e desvio-padrão do comprimento axial médio $(\mathrm{mm})$ em cães, segundo o grupo

\begin{tabular}{ccccc}
\hline Grupo & Olho operado & $\begin{array}{c}\text { Olho não } \\
\text { operado }\end{array}$ & Média & $\begin{array}{c}\text { Análise de perfil } \\
\text { P-valor }\end{array}$ \\
\hline G1 & $19,71 \pm 1,15$ & $19,37 \pm 1,08$ & $19,54 \pm 1,10$ & 0,656 \\
G2 & $20,19 \pm 1,09$ & $20,44 \pm 0,92$ & $20,33 \pm 1,03$ & 0,590 \\
\hline Média & $19,97 \pm 1,17$ & $19,91 \pm 1,11$ & $19,94 \pm 1,12$ & 0,116
\end{tabular}

G1: cães submetidos à facoemulsificação com implante de lentes intra-oculares em piggyback; G2: cães submetidos à facoemulsificação.

Não houve diferença entre os olhos $(\mathrm{P}>0,05)$ direito e esquerdo.

Tabela 3. Média e desvio-padrão referentes à ceratometria (dioptria) em cães, segundo o grupo e o momento

\begin{tabular}{cccccc} 
Grupo & M1 & M2 & M3 & Média & $\begin{array}{c}\text { Análise de perfil } \\
\text { P- valor }\end{array}$ \\
\hline G1 & $41,09 \pm 2,49$ & $41,25 \pm 2,39$ & $41,18 \pm 2,54$ & $41,18 \pm 2,36$ & 0,677 \\
G2 & $40,38 \pm 2,71$ & $40,85 \pm 2,55$ & $40,85 \pm 2,61$ & $40,69 \pm 2,54$ & 0,604 \\
\hline Média & $40,69 \pm 2,56$ & $41,03 \pm 2,41$ & $41,00 \pm 2,51$ & $40,93 \pm 2,45$ & 0,838
\end{tabular}

G1: cães submetidos à facoemulsificação com implante de lentes intra-oculares em piggyback; G2: cães submetidos à facoemulsificação. M1: pré-cirurgia; M2: 30 dias pós-cirurgia; M3: 60 dias pós-cirurgia.

Não houve diferença entre grupos dentro de momentos e entre momentos dentro de grupos.

A refração média aferida no M3 dos olhos operados do grupo-controle (G2) foi de $19,47 \pm 1,13 \mathrm{D}$, variando entre $21,25 \mathrm{D}$ e $18,25 \mathrm{D}$. No grupo com o implante de lente intra-ocular (G1), este valor foi de $5,57 \pm 1,59 \mathrm{D}$, com o máximo de 7 e o mínimo de 3D. Não foi possível a aferição da refração em M1, devido à presença de catarata e, em M2, devido ao fato de os meios refrativos '1não estarem completamente transparentes. A refração não foi aferida em dois animais do G1 devido à presença de opacidades intra-oculares e formação de sinéquia posterior; em um destes, o olho desenvolveu glaucoma, impossibilitando a realização da retinoscopia em faixa.

Houve diferença significativa $(\mathrm{P}<0,001)$ entre os animais dos dois grupos.

\section{DISCUSSÃO}

O cálculo do poder da LIO depende de seis variáveis: erro refrativo pós-operatório desejado, poder córneo médio, fator de profundidade da câmara anterior (constante-A), fórmula de cálculo da LIO, qualidade da LIO 
(responsabilidade do fabricante) e, mais importante, comprimento axial do olho. A determinação precisa da emetropia pósoperatória depende da minimização dos erros associados à mensuração destes parâmetros (Krag e Olsen, 1991; Hoffer, 1994; Rajan et al., 2002; Goyal et al., 2003).

O erro refrativo almejado no presente trabalho foi o de zero dioptria, porém nenhum dos animais com implante de lente intra-ocular apresentou como resultado a emetropia desejada. Os valores variaram de 3 a 7D. Tromans et al. (2001), em um trabalho com pacientes humanos pediátricos, mostraram que o erro refrativo estimado em olhos de comprimento axial menor que $20 \mathrm{~mm}$, usando a fórmula $\mathrm{SRK} / \mathrm{T}$, diferiu, em alguns casos, do erro refrativo pós-operatório, em mais de 9,19D.

Um dos fatores que contribui para a diferença entre o erro refrativo estimado e o pós-operatório é a mensuração incorreta do poder refrativo corneal aferido por ceratômetros e sistemas de topografia corneal (Aramberri, 2003). Nesta pesquisa, verificou-se córnea discretamente mais plana $(40,69 \pm 2,56 \mathrm{D})$ e astigmatismo de $0,68 \pm 0,56 \mathrm{D}$, o que levaria ao cálculo de uma lente intra-ocular mais potente, valores estes próximos aos obtidos por Gaiddon et al. (1991), de $39,94 \pm 2,61 \mathrm{D}$, com pequeno grau de astigmatismo.

A média do poder corneal tem pouca mudança após a cirurgia de catarata por facoemulsificação, porém um pequeno achatamento corneal pode ocasionar erros refrativos pós-cirúrgicos (Olsen, 1992; Brandser et al., 1997; Kora et al., 2001). Hayashi et al. (1995), Lyle e Jin (1996) e Masket e Tennen (1996) mostraram que incisões em córnea clara de $3,2 \mathrm{~mm}$, sem a presença de suturas, tiveram maior estabilidade córnea, causando astigmatismo de, no máximo, $0,5 \mathrm{D}$. No presente estudo, apesar da aplicação de um ou dois pontos, o valor de astigmatismo observado não foi estatisticamente significante.

$\mathrm{O}$ efeito refrativo da lente intra-ocular depende, também, de sua posição dentro do olho. Se o implante for posicionado mais próximo à córnea, a refração irá tender à miopia, e se for mais distante, o olho ficará mais hipermétrope. A estimativa incorreta da profundidade da câmara anterior (PCA), pós-cirúrgica, corresponde a
42\% dos erros no cálculo da LIO (Olsen, 1992). Assim, devido à maior distância entre a córnea e o centro da lente canina, a PCA pós-operatória foi maior, contribuindo para um erro refracional

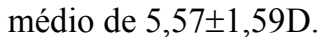

Hoffer (1993) e Kijima et al. (1999) obtiveram bons resultados em olhos longos $(>26 \mathrm{~mm}$ e $>27 \mathrm{~mm}$, respectivamente), utilizando a fórmula SRK/T. Tromans et al. (2001) e Eleftheriadis (2003) demonstraram que, em olhos com comprimento axial menor que $20 \mathrm{~mm}$, altos erros do poder dióptrico da lente podem ocorrer. Segundo Retzlaff et al. (1990), na fórmula $\mathrm{SRK} / \mathrm{T}$, há descrição de $46 \%$ de probabilidade de os casos serem corrigidos corretamente dentro de $0,5 \mathrm{D}, 66 \%$ dentro de $1 \mathrm{D}$ e $2 \%$ maior do que $2 \mathrm{D}$ em olhos curtos $(<22 \mathrm{~mm})$.

Erros na mensuração do comprimento axial promovem grande influência nas previsões de emetropia pós-operatória, podendo resultar em $54 \%$ a $68 \%$ dos desvios na refração pósoperatória (Olsen, 1992; Goyal et al., 2003). No presente trabalho, o valor médio de comprimento axial foi de 19,94 $\pm 1,12 \mathrm{~mm}$, e o dos olhos que receberam o implante intra-ocular $19,71 \pm 1,15 \mathrm{~mm}$, semelhante aos valores descritos por Gaiddon et al. (1991), Olsen (1992) e Goyal et al. (2003). Soares (2002), ao utilizar cães da raça Fila Brasileiro, relatou comprimento axial médio de 22,15 $\pm 0,49 \mathrm{~mm}$, e Sampaio (2000), ao avaliar animais de pesos e sexos diferentes, obteve o valor máximo de $20,40 \mathrm{~mm}$, mostrando que o comprimento axial do olho de cães é semelhante ao do olho humano curto $(<22 \mathrm{~mm})$.

A utilização de duas lentes intra-oculares em piggyback promove o deslocamento posterior da lente mais próxima à cápsula posterior e leve aplanação na região de contato entre as duas lentes intra-oculares, diminuindo o seu poder dióptrico efetivo e contribuindo para o alto erro refrativo final (Oshika et al., 2001; Spencer et al., 2002).

A biometria ultra-sônica é um método preciso para mensuração das distâncias oculares, especialmente na determinação do comprimento axial e dos segmentos oculares para o cálculo do poder da lente intra-ocular (Haigis, 2000). Um erro de $100 \mu \mathrm{m}$ no comprimento axial pode levar 
a um erro refracional de 0,28D (Olsen, 1987; Eleftheriadis, 2003).

Um dos fatores que propiciam erro na mensuração do comprimento axial ocorre devido à diferença entre a biometria de modo-A de contato e a de imersão (Kora et al., 2001). O ultra-som de contato causa indentação da córnea e, conseqüentemente, diminuição na mensuração do comprimento axial em relação à de imersão, na qual a sonda do ultra-som é colocada em solução, nunca entrando em contato com a córnea (Goyal et al., 2003). Segundo Olsen e Nielsen (1989), a técnica de contato apresentou diferença de $0,14 \mathrm{~mm}$ no comprimento axial em relação à técnica de imersão, portanto, $0,5 \mathrm{D}$ de erro no poder final da LIO.

A velocidade do ultra-som também pode resultar na mensuração inadequada do comprimento axial. O ecobiômetro não mede o comprimento diretamente, e, sim, o tempo em microssegundos que leva para a onda de som percorrer o trajeto da córnea para a retina (Hoffer, 1994; Kora et al., 2001). A velocidade média mais utilizada é de $1550 \mathrm{~m} / \mathrm{s}$, mas, em olhos pequenos, a lente representa uma proporção maior do comprimento axial total e, deste modo, a velocidade média deve ser mais rápida (Tromans et al., 2001). Erros também podem ocorrer devido à dificuldade de se determinar, com precisão, a velocidade do ultra-som em cataratas de diferentes densidades e na correta determinação da distância do vítreo à retina (Olsen, 1992; Brandser et al., 1997). A velocidade utilizada foi a mesma descrita por Schiffer et al. (1982) e Davidson e Nelms (1999), de 1,526 m/s para o humor aquoso e o vítreo, e de $1,710 \mathrm{~m} / \mathrm{s}$ para o cristalino. $\mathrm{O}$ erro na mensuração da distância do vítreo à retina ocorre devido ao fato de o ultrasom ser refletido a partir da membrana limitante interna, que não corresponde à verdadeira espessura retiniana. Fórmulas para o cálculo de LIO de terceira geração levam em conta esta espessura, introduzindo um fator retiniano de correção (Goyal et al., 2003).

Talvez com o cálculo de uma constante-A personalizada e optimizando a fórmula SRK/T com dados próprios, melhores resultados pudessem ser obtidos, visto que os olhos dos cães, principalmente os das raças mais predispostas ao desenvolvimento de catarata (Poodle, Schnauzer Miniatura, Cocker Spaniel
Americano, entre outros), de porte pequeno e médio, apresentam comprimento médio axial menor e profundidade de câmara anterior e cristalino maiores do que os olhos humanos.

\section{CONCLUSÃO}

$\mathrm{O}$ estudo demonstrou que a fórmula $\mathrm{SRK} / \mathrm{T}$ não foi adequada para a reabilitação visual de cães cujo objetivo final era a emetropia. O erro refracional atingido não correspondeu às expectativas ao se utilizar uma fórmula de cálculo de lente intra-ocular de terceira geração, mesmo minimizando os possíveis erros na mensuração dos dados utilizados pela fórmula.

\section{REFERÊNCIAS BIBLIOGRÁFICAS}

ARAMBERRI, J. Intraocular lens power calculation after corneal refractive surgery: Double-K method. J. Cataract Refract. Surg., v.29, p.2063-2068, 2003.

BISTNER, S.I. Recent developments in comparative ophthalmology. Compend. Cont. Educ. Pract. Vet., v.14, p.1304-1323, 1992.

BRANDSER, R.; HAASKJOLD, E., DROLSUM, L. Accuracy of IOL calculation in cataract surgery. Acta Ophthalmol. Scand., v.75, p.162-165, 1997.

DAVIDSON, M.G.; MURPHY, C.J.; NASISSE, M.P. et al. Refractive state of aphakic and pseudophakic eyes of dog. Am. J. Vet. Res., v.54, p.174-177, 1993.

DAVIDSON, M.G.; NELMS, S.R. Diseases of the lens and cataract formation. In: GELATT, K.N. Veterinary ophthalmology. 3.ed. Philadelphia: Lippincot Williams \& Wilkins, 1999. p.797-825.

ELEFTHERIADIS, H.; SCISCIO, A.; ISMAIL, A. et al. Primary polypseudophakia for cataract surgery in hypermetropic eyes: refractive results and longterm stability of the implants within the capsular bag. Br. J. Ophthalmol., v.85, p.1198202, 2001.

ELEFTHERIADIS, H. IOL Master biometry: refractive results of 100 consecutive cases. $\mathrm{Br} . \mathrm{J}$. Ophthalmol., v.87, p.960-963, 2003.

ERKIN, E.F.; TARHAN, S.; ÖZTÜRK, F. Axial length measurement and asteroid hyalosis. $J$. 
Cataract Refract. Surg., v.25, p.1400-1403, 1999.

FISCHER, C.A. Geriatric ophthalmology. Vet. Clin. N. Am.: Small Anim. Pract., v.19, p.103123, 1989.

GAIDDON, J.; ROSOLEN, S.G.; STERU, L. et al. Use of biometry and keratometry for determining optimal power for intraocular lens implants in dogs. Am. J. Vet. Res., v.52, p.781783, 1991.

GIERS, U.; EPPLE, C. Comparison of A-scan device accuracy. J. Cataract Refract. Surg., v.16, p.235-242, 1990.

GLOVER, T.D.; CONSTANTINESCU, G.M. Surgery for cataract. Vet Clin. N. Am.: Small Anim. Pract., v.27, p.1143-1173, 1997.

GOYAL, R.; NORTH, R.V.; MORGAN, J.E. Comparison of laser interferometry and ultrasound A-scan in the measurement of axial length. Acta Ophthalmol. Scand., v.81, p.331335, 2003.

HAIGIS, W.; LEGE, B.; MILLER, N. et al. Comparison of immersion ultrasound biometry and partial coherence interferometry for intraocular lens calculation according to Haigis. Graefe's Arch. Clin. Exp. Ophthalmol., v.238, p.765-773, 2000.

HAYASHI, K.; HAYASHI, H.; NAKAO, F. et al. The correlation between incision size and corneal shape changes in sutureless cataract surgery. Ophthalmology, v.102, p.550-556, 1995.

HOFFER, K.J. The Hoffer Q formula: a comparison of theoretic and regression formulas. J. Cataract Refract. Surg., v.19, p.700-712, 1993.

HOFFER, K.J. Ultrasound velocities for axial eye length measurement. J. Cataract Refract. Surg., v.20, p.554-562, 1994.

HULL, C.C.; LIU, C.S.C.; SCISCIO, A. Image quality in polypseudophakia for extremely short eyes. Br. J. Ophthalmol., v.83, p.656-663, 1999.

JAFFE, N.S.; JAFFE, M.S.; JAFFE, G.F. Intraocular lens implants. In:__. Cataract Surgery and its Complications. 6.ed. St. Louis: Mosby Company, 1997. p.147-199.

KIJIMA, T.; KOZAWA, T.; KORA, Y. et al. Accuracy of intraocular power calculation formulas. J. Jpn. Ophthalmol. Soc., v.103, p.470476, 1999.

KORA, Y.; SHIMIZU, K.; YOSHIDA, M. et al. Intraocular lens power calculation for lens exchange. J. Cataract Refract. Surg., v.27, p.543-548, 2001.

KRAG, S.; OLSEN, T. Secondary IOL power calculation - a comparison of an optical and a biometric method. Acta Ophthalmol., v.69, p.625-629, 1991.

LAGENBUCHER, A.; SEITZ, B. Computerized calculation scheme for toric intraocular lenses. Acta Ophthalmol. Scand., v.82, p.270-276, 2004.

LYLE, W.A.; JIN, G.J.C. Prospective evaluation of early visual and refractive effects with small clear corneal incision for cataract surgery. $J$. Cataract Refract. Surg., v.22, p.1456-1460, 1996.

MASKET, S.; TENNEN, D.G. Astigmatic stabilization of $3.0 \mathrm{~mm}$ temporal clear corneal cataract incisions. J. Cataract Refract. Surg., v.22, p.1451-1455, 1996.

MURPHY, C.; TUFT, S.J.; MINASSIAN, D.C. Refractive error and visual outcome after cataract extraction. J. Cataract Refract. Surg., v.28, p.6266, 2002.

NARFSTRÖM, K.; BJERKÅS, E. Visual Impairment. In: PEIFFER Jr., R.L.; PETERSENJONES, S.M. Small animal ophthalmology: A problem-oriented approach. 3.ed. London: Harcourt Publishers, 2001. p.103-176.

OLSEN, T. Theorical approach to intraocular lens calculation using Gaussian optics. $J$. Cataract Refract. Surg., v.13, p.141-145, 1987.

OLSEN, T. Sources of error in intraocular lens power calculation. J. Cataract Refract. Surg., v.18, p.125-129, 1992.

OLSEN, T.; NIELSEN, P.J. Immersion versus contact technique in the measurement of axial length by ultrasound. Acta Ophthalmol., v.67, p.101-102, 1989.

OSHIKA, T.; IAMAMURA, A.; AMANO, S. et al. Piggyback foldable intraocular lens implantation in patients with microphthalmos. $J$. Cataract Refract. Surg., v.27, p.841-844, 2001.

PREUSSNER, P.R.; WAHL, J.; LAHDO, H. et al. Ray for intraocular lens calculation. $J$. 
Cataract Refract. Surg., v.28, p.1412-1419, 2002.

RAJAN, M.S.; KEILHORN, I.; BELL, J.A. Partial coherence laser interferometry vs. conventional ultrasound biometry in intraocular lens power calculations. Eye, v.16, p.552-556, 2002.

RANZANI, J.J.T.; MOBRICCI, L.A.L.; CARVALHO, L.R.; BRANDÃO, C.V.S. Refração por retinoscopia com luz em faixa em cães. Arq. Bras. Med. Vet. Zootec., v.60, p.353355, 2008.

RETZLAFF, J.A.; SANDERS, D.R.; KRAFF, M.C. Development of the SRK/T intraocular lens implant power calculation formula. J. Cataract Refract. Surg., v.16, p.333-340, 1990.

RODRIGUES, G.N. Estudo clínico da facoemulsificação em cães, com e sem implante de lente intra-ocular em piggyback. Botucatu, 2004. 194f. Tese (Doutorado) - Faculdade de Medicina Veterinária e Zootecnia, Universidade Estadual Paulista, Botucatu.

SAMPAIO, G.R. Influência dos parâmetros sexual, anatômico e peso sobre mensurações oculares e cálculo de poder dióptrico de lentes intra-oculares no cão. 2000. 187f. Dissertação (Mestrado) - Faculdade de Medicina Veterinária e Zootecnia, Universidade Estadual Paulista, Botucatu.

SCHIFFER, S.P.; RANTANEN, N.W.; LEARY, G.A. et al. Biometric study of the canine eye, using A-mode ultrasonography. Am. J. Vet. Res., v.43, p.826-830, 1982.

SLATTER, D.H. Lens. In: SLATTER, D.H. Fundamentals of veterinary ophthalmology. 3.ed. Philadelphia: W.B. Saunders, 2001. p.381-410.

SOARES, A.M.B. Ceratometria, retinoscopia e ecobiometria do bulbo do olho de cães da raça Fila Brasileiro. 2002. 61f. Tese (Doutorado) Faculdade de Ciências Agrárias e Veterinárias, Universidade Estadual Paulista, Jaboticabal.

SPENCER, T.S.; MAMALIS, N.; LANE S.S. Interlenticular opacification of piggyback acrylic intraocular lenses. J. Cataract. Refract. Surg., v.28, p.1287-1290, 2002.

TROMANS, C.; HAIGH, P.M.; BISWAS, S. et al. Accuracy of intraocular lens power calculation in pediatric cataract surgery. $\mathrm{Br} . \mathrm{J}$. Ophthalmol., v.85, p.939-941, 2001. 